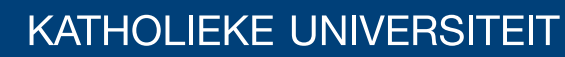 \\ LEUVEN
}

\section{Faculty of Business and Economics}

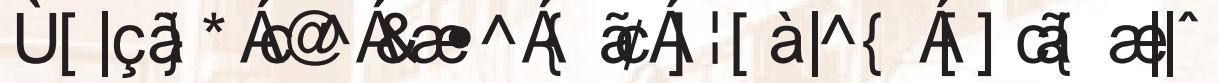
EI IXMQJIELDQRKIDQGGSUFHDDORULKP V

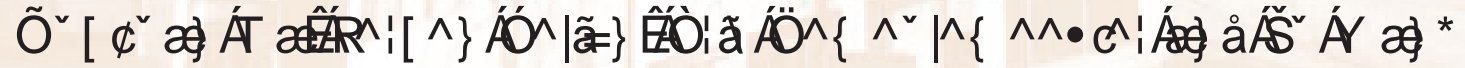

DEPARTMENT OF DECISION SCIENCES AND INFORMATION MANAGEMENT (KBI) 


\title{
Solving the case mix problem optimally by using branch-and-price algorithms
}

\author{
Guoxuan $\mathrm{Ma}^{1}$, Jeroen Beliën ${ }^{1,2}$, Erik Demeulemeester ${ }^{1}$, Lu Wang $^{1}$ \\ ${ }^{1}$ Katholieke Universiteit Leuven, Faculty of Business and Economics \\ Department of Decision Sciences and Information Management \\ Naamsestraat 69, B-3000 Leuven, Belgium \\ Tel: +32-16-32.69.72 \\ Fax: +32-16-32.66.24 \\ guoxuan.ma@econ.kuleuven.be \\ jeroen.belien@econ.kuleuven.be \\ erik.demeulemeester@econ.kuleuven.be \\ lu.wang@econ.kuleuven.be \\ ${ }^{2}$ Hogeschool-Universiteit Brussel, Campus Economische Hogeschool \\ Centrum voor Modellering en Simulatie \\ Stormstraat 2, B-1000 Brussel, Belgium \\ jeroen.belien@hubrussel.be
}




\section{Solving the Case Mix Problem Optimally by Using Branch-and-price Algorithms}

This paper describes a methodology for the case mix problem in the health care sector. Aiming at maximizing the overall financial contribution of the given resource capacity within a hospital, the case mix problem is formulated as an integer linear programming model to produce the optimal patient mix pattern together with its associated resource allocation scheme. In order to solve the huge integer program optimally, an efficient solution approach, branch-and-price, is proposed, developed and implemented in this research. When studied from the column generation perspective, the integer linear programming model can be formulated differently. According to different decomposition units, namely wards, surgeon groups and patient groups, three decomposition based reformulations are built respectively. Among them, the first two reformulations are suitable to be solved within the framework of branch-and-price, while this is not the case for the last one. Numerical experiments are carried out, and the computational results are presented and compared, which demonstrate that the branch-and-price approach outperforms the integer linear programming method significantly and that decomposition on wards performs much better than decomposition on surgeon groups both with respect to the solution quality and the computation speed.

Key words: patient case mix; capacity planning; integer linear programming; branch-and-price approach 


\section{Introduction}

The performance of the health care industry has a strong impact on the life of human beings. Hence, its efficiency and effectiveness are always of great concern in the field of health care management. However, the complexity within health care systems typically makes the efficient management of hospitals quite difficult, forcing professionals to deal with the complex problems of hospitals' production and operations systematically through using advanced methods and models of operational research and the management sciences. As pointed out by Carter (2002), these complex problems in the health care sector could benefit from operations research-type analysis and applications.

In order to apply the traditional operations research methods to hospital management, many studies have developed essential concepts and practical frameworks. Rhyne and Jupp (1988) and Roth and Van Dierdonck (1995) both suggest the application of the manufacturing resource planning system to health care organizations, and adopt the concept of diagnosis related groups (DRGs) (Fetter and Freeman 1986) to define health services as the hospital's products. Based on the applicability, a new operations planning and control system is developed for the health service production. Moreover, Vissers et al. (2001) present a hierarchical framework for the production control of hospitals, which consists of five levels of planning and control on the basis of the decisions made at different levels. At each level of the framework, sound decisions have to be made regarding patient flows and hospital resources in order to balance the trade-offs between service and efficiency, and the coordination of various planning levels is linked by the control functions required for the hospital production management.

The case mix problem in the health care sector involves the long- and medium-term decisions that are taken both at the patient volumes planning and control level and at the resources planning and control level of the production control framework of Vissers et al. (2001). These decisions made in a hospital are concerned with the number of patients of different pathologies that can be treated and the corresponding resource capacity requirements, which are strongly dependent on the trade-offs between the capacity provided and patient demands at each department of the hospital. Here, the case mix (Fetter et al. 1980) is 
a critical decision variable representing the number and mix of patients of various patient groups, and the patient grouping can be based on the concept of DRGs (Fetter and Freeman 1986) or follow a procedure of an iso-process grouping rule (Vissers et al. 2001). Hospitals may choose the patient case mix according to different targets and policies under the given resource capacity, so that the case mix problem in the health care sector can be comparable with the product mix decision problem within a manufacturing system (Dowling 1976; Robbins and Tuntiwongpiboon 1989).

Generally, hospitals can be differentiated into two categories: constrained profit satisfiers and profit maximizers (Simon 1960), dependent on the opposite goals of health service providers. Constrained profit satisfier hospitals are assumed to be motivated by professional interests rather than economic returns. The target of profit satisfiers is to adhere to the preferred case mix pattern or to meet other preset objectives on condition that they can manage to break even (or preserve their previous income level) without violating the capacity constraints (Rifai and Pecenka 1990; Blake and Carter 2002). On the other hand, the profit maximizer hospitals are assumed to be positioned in a competitive market environment and thus they are prone to choose patient cases that will bring maximum rewards within the given capacity. Actually, many studies have explored the hospital's financial topics, such as Brandeau and Hopkins (1984), Dexter et al. (2002a; 2002b), Hatcher and Connelly (1988) and Mulholland et al. (2005).

In the context of this research, the hospital is assumed to be a profit maximizer in order to cope with the increasing economic pressures in the health care sector (OECD 2010). Basically, given the predictions of the number of patients that will enter a hospital with a certain pathology, given the expected financial contribution, the expected surgery time and the expected length of stay per pathology and given the knowledge to which surgeon group and to which ward a patient with a certain pathology will be assigned, the case mix problem in this study is to determine how many patients from each pathology group can be taken care of at the hospital over the course of one year in view of maximizing the overall contributions, as well as accordingly to determine how operating rooms are assigned to surgeon groups and how many beds are allocated to each ward. As a result, an optimal patient case mix pattern generating maximum profits is sought, accompanied by a time-phased allocation scheme of resources. 
The literature on the case mix problem is relatively scarce. Boldy (1976) comments on a number of potentially very valuable studies on the application of mathematical programming methods to the case mix problems. Linear programming models are firstly proposed to determine the mix and volume of different types of patients (Feldstein 1967; Baligh and Laughhunn 1969; Dowling 1976; Hughes and Soliman 1985; Robbins and Tuntiwongpiboon 1989). Goal programming is another branch of methods seeking to solve the case mix decision for the hospital with multiple objectives (Rifai and Pecenka 1989; Blake and Carter 2002). Also, integer linear programming models (Adan and Vissers 2002; Vissers et al. 2005) are recently developed to generate patient admission profiles. Besides, mathematical programming methods are also widely applied to the capacity allocation problems in the health care sector (Tingley and Liebman 1984; Blake et al. 2002; Blake and Donald 2002; Kuo et al. 2003).

However, Smith-Daniels et al. (1988) present an extensive literature review on capacity management at hospitals and indicate that most admission scheduling systems only consider bed capacity, which may result in sub-optimization in the utilization of other resources. Gemmel and Van Dierdonck (1999) point out that there exists a large gap between the theoretical models and the practical applications of patient admission systems. Vissers et al. (2007) illustrate that the current concept of hospital admission planning only emphasizes the utilization of scarce resources but neglects the level of services offered to patients. Hence, it becomes of necessity to investigate the trade-offs between patient mix and resource efficiency as well as the utilization of multiple resources from the perspective of a whole hospital.

In this research, the case mix planning problem within a hospital is studied intensively. Taking into account the given capacity of multiple resources, including operating rooms and beds, hospitals choose the optimal patient case mix with the objective of maximizing the resource efficiency, i.e., the overall financial contribution. A mathematical modeling is built for the case mix problem, and effective branchand-price algorithms are designed to solve the integer program model optimally.

This paper is structured as follows. Section 2 describes the case mix problem in detail and formulates it as an integer linear programming model. In Section 3, the model is interpreted from the perspective of column generation, and three reformulations are built on the basis of the concept of decomposition. Then, 
the branch-and-price algorithms are developed completely. After that, numerical experiments are carried out to evaluate the efficiency and effectiveness of the designed algorithms, and the computational results are presented and analyzed comprehensively in Section 4. Finally, Section 5 draws the conclusions of this paper and suggests potential areas for further research.

\section{Problem Statement}

In this research, the health care provider (i.e., hospital) is assumed to be a profit maximizer, which intends to select an optimal case mix generating maximum profits under the provided resource capacity and other certain constraints. It is assumed that there is reserved capacity for emergency patients at the hospital. Hence, only elective surgery cases are considered, while emergencies are left out of the case mix planning. Additionally, it is noteworthy that the "profit" is not limited to the financial contribution, but may refer to other desired performance indicators, such as the priority score of particular pathologies.

In this section, the case mix problem is elaborated through a series of explanations and definitions firstly, and then a mathematical model is developed in detail. In order to define the health care settings, the following observations and assumptions are made accordingly:

1) A hospital is supposedly divided into a number of different business units (i.e., departments). Each department is considered as an independent specialized unit, which consists of a few surgeon groups and its own ward with a fixed number of beds to serve the patients entering this unit. Besides the departments, a public surgical center with a few operating rooms (ORs) is assumed to be located in the hospital. Surgeon groups, operating rooms and hospital beds are the main resources consumed by patients in our configuration. Other resources, such as nursing staffs and intensive care beds, are not covered in this research.

2) Each surgeon group is assumed to be specialized in one medical field, which may cover a few similar pathology types, and usually contains a certain number of equally qualified surgeons, who are interreplaceable when performing surgery on their patients. 
3) The surgical center is assumed to be open during work days, i.e., from Monday till Friday, which are called active days in our description, but to be closed in the weekend, i.e., Saturday and Sunday being named inactive days. Operating rooms are shared by all surgeon groups of the hospital but are allotted to each surgeon group periodically. Once an operating room is assigned to a surgeon group for a certain period, it will be used exclusively by this surgeon group. The concept of an operating block is defined as the smallest time unit for which an operating room can be assigned to a surgeon group performing surgeries. The block length is typically defined as eight hours, which means that each operating room will be occupied by at most one surgeon group during an active day, but this block length can be easily changed into other unit lengths (e.g., four hours) if needed. Finally, it should be mentioned that the special surgery cases with a planned duration that is longer than the block length will be excluded from our discussion.

4) Different from operating rooms, hospital beds are available during a whole week. In contrast with the time-phased allocation of OR blocks, beds are assigned to each ward in a fixed pattern. Theoretically, a flexible bed allocation schedule that allows bed sharing will help to level the bed occupancy among various wards and thus boost the overall utilization of the bed capacity. However, the bed allocation decisions are usually made in the tactical planning phase and will remain constant over a certain period before being readjusted. Thus, in our assumption, beds that are allocated to a ward are only reserved to accommodate patients from the corresponding department.

5) Practically, a patient is delineated by a number of characteristics. The first one is the surgeon group that can perform surgery on the patient (remark: one surgeon group may treat several pathology types, but patients with a certain pathology can only be treated by one surgeon group). The second one is the OR time that is occupied by the patient, which is called the surgery duration. The third one is the number of days for which the patient seizes a bed for recovery in the corresponding ward, namely the length of stay (LOS), and the last one is a parameter denoting the financial return of treating the patient, i.e., the reward of the treatment. According to the above introduced characteristics, patients are distinguished into various iso-process groups (Vissers et al. 2002). 
6) In reality, the surgery duration and/or the LOS of every patient within one group may fluctuate to some extent due to their individual physical status. However, because of the mathematical modeling, we assume these parameters to be deterministic and identical for all patients within the same group. Thus, a patient group is defined such that patients within one group all have the same parameters but those from different groups differ from each other for at least one of the parameters above.

7) Generally speaking, patients are assumed to be admitted in the morning of the day of their surgery, and then seize a bed consecutively until they leave the hospital on the last day of their hospitalization. Their hospitalization is counted from the day patients enter the hospital and lasts for their respective LOS. Specifically, patients receive surgery from a dedicated surgeon group on the admission day, get transferred to the corresponding ward subsequently and then stay for a certain number of days to get recovery. See Figure 1 for an illustration of the relationship among wards, surgeon groups and patient groups within a hospital.

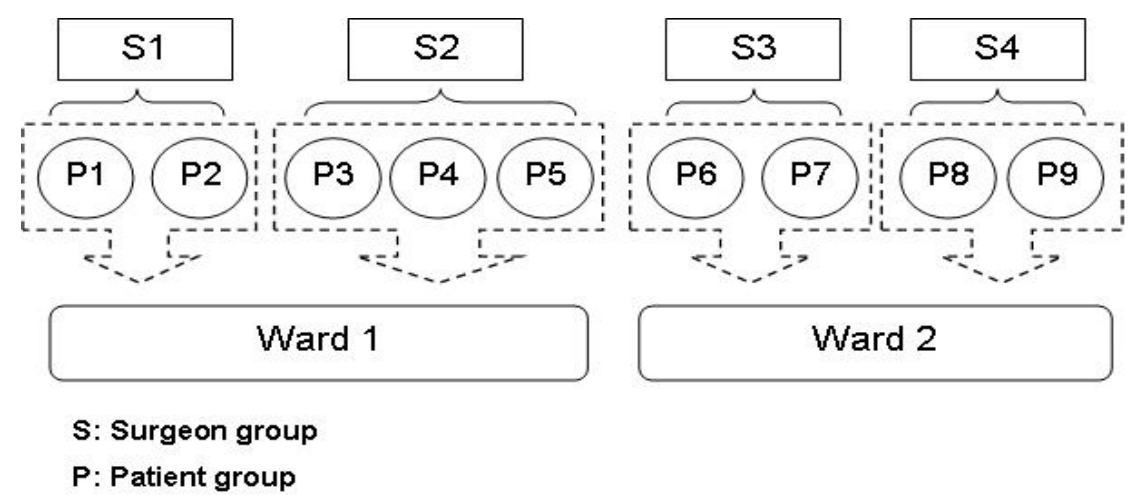

Figure 1: Relationship among Wards, Surgeon Groups and Patient Groups

8) A case mix plan is effective for six months to one year. We assume that this period consists of a certain number of repeating cycles with a cycle length of one week, which can be easily extended to other cycle lengths according to the actual situation.

9) The number of patients of each patient group that can undergo surgery during a cycle period is constrained by various factors, such as the existing resource capacity, the patient arrivals or the minimum patient admission level mandated by the health care authorities or insurance agencies, and so on. For instance, the surgeon capacity sets an upper limit for the maximum number of patients that 
can be treated periodically. Thereby, for each patient group a numerical range is specified for the total number of patients that can get admitted during one cycle length.

In addition, in order to build a mathematical model for the case mix problem, the required notations are defined below.

The adopted sets and indices consist of:

$W$ : set of wards with index $w$;

$S$ : set of surgeon groups with index $s$;

$P$ : set of patient groups with index $p$;

$D$ : set of days within a cycle with index $d$;

$A$ : set of active days within a cycle with index $a$;

$S_{w}$ : subset of surgeon groups $S$ whose patients are moved to ward $w$ after surgery is performed;

$P_{w}$ : subset of patient groups $P$ whose patients occupy the beds of ward $w$;

$P_{s}$ : subset of patient groups $P$ whose patients are operated on by surgeon group $s$;

$A_{p d}$ : subset of active days $A$ for which it applies that patients of group $p$ still occupy a bed on day $d$ after having undergone surgery on these days. (Remark: for the patient group $p$ with an average LOS being longer than the cycle length, an active day a may be counted multiple times.)

The needed coefficients and the right hand side values include:

$r_{p}$ : the profit generated by treating a patient of patient group $p$

$d u r_{p}$ : the surgery duration time of operating on a patient of patient group $p$;

$L B_{p}$ : the lower bound on the number of patients of patient group $p$ that can be treated per cycle;

$U B_{p}$ : the upper bound on the number of patients of patient group $p$ that can be treated per cycle;

$B E D S$ : the total number of available beds (assumed to be the existing bed capacity);

BLOCKS: the total number of available OR blocks (assumed to be the existing block capacity); LENGTH: the operating room block length (assumed to be 480 minutes).

The decision variables comprise: 
$x_{p a}:$ the number of patients of patient group $p$ that receive surgery on active day $a$;

$y_{w}:$ the number of beds allocated to ward $w$;

$z_{s a}:$ the number of OR blocks assigned to surgeon group $s$ on active day $a$.

Then, with the assumptions and notations given above, the case mix problem that aims at maximizing the overall hospital profits is formulated as an integer linear programming (ILP) model:

$$
\begin{array}{ll}
\max & \sum_{p \in P} r_{p} \sum_{a \in A} x_{p a} \\
\text { s.t. } & \sum_{w \in W} y_{w} \leq B E D S, \\
& \sum_{p \in P_{w}} \sum_{a \in A_{p d}} x_{p a} \leq y_{w}, \quad \forall w \in W, d \in D, \\
& \sum_{s \in S} z_{s a} \leq B L O C K S, \quad \forall a \in A, \\
& \sum_{p \in P_{S}} d u r_{p} x_{p a} \leq z_{s a} L E N G T H, \quad \forall s \in S, a \in A, \\
& L B_{p} \leq \sum_{a \in A} x_{p a} \leq U B_{p}, \quad \forall p \in P, \\
& x_{p a}, y_{w}, z_{s a} \in \mathbb{Z}^{+}, \quad \forall w \in W, s \in S, p \in P, a \in A .
\end{array}
$$

The objective function (1) maximizes the sum of the generated profits from treating all patient groups during one cycle. Constraint (2) regulates the allocation of the bed capacity. Constraint set (3) ensures that the daily bed occupancy of each ward cannot exceed its allocated capacity. Constraint set (4) prescribes the time-phased assignment of OR blocks. Constraint set (5) reflects that every surgeon group has enough assigned blocks to operate on its scheduled patients on each active day. Constraint set (6) defines for each patient group a numerical range into which the number of admitted patients should fall within a cycle. Constraint (7) indicates that all decision variables are nonnegative integers.

Generally, the above formulation results in a huge integer program even for a comprehensive hospital of regular size (e.g., 25 departments). Specifically, considering a hospital with $l$ wards, $m$ surgeon groups 
and $n$ patient groups, the ILP modeling produces $l+5 m+5 n$ decision variables. Thus, for the instance of a hospital possessing 25 departments, each of which is assumed to have three surgeon groups and each surgeon group can treat three patient groups on average, the formulated ILP model contains 1525 integer variables. Basically, no commercial ILP software (e.g., ILOG CPLEX) can solve this integer problem for realistic dimensions efficiently, which motivates us to propose the more powerful solution approach, i.e., the branch-and-price, to cope with this huge integer program (Barnhart et al. 1998).

\section{The Branch-and-price Approach}

In this section, the branch-and-price approach is elaborated in three steps. First, the ILP model of the case mix problem is interpreted from the column generation perspective (Winston 1997; Barnhart et al. 1998), such that the constructed reformulation is suitable to be solved within the framework of branch-and-price. Second, the branch-and-price algorithm is created completely through specifying key steps. Finally, a few acceleration techniques are addressed to improve the designed algorithms. Actually, there already exist a number of previous works that successfully applied a branch-and-price approach to a hospital planning or scheduling problem (e.g., Bard and Purnomo 2005; Beliën and Demeulemeester 2006, 2007, 2008).

\subsection{Decomposition Based Reformulation}

When studied from the perspective of column generation, the ILP model of the case mix problem can be reformulated differently. In fact, although the decision variables $y_{w}$ and $z_{s a}$ reflect the different levels of resource rationing (beds and OR blocks respectively), $x_{p a}$ is the fundamental driving force of the resource assignment. Once the number of patients of group $p$ to be treated on day $a$ is determined for all patient groups and for all active days within a cycle, the bed demands of each ward $\left(y_{w}\right)$ and the daily block requirements of each surgeon group $\left(z_{s a}\right)$ are obtained accordingly. Instead of deciding the value of $x_{p a}$ on an individual basis, we can aggregate the decisions for all active days within a cycle. This means that the decision variable $x_{p a}$ can be replaced by a new decision variable that is derived from a set of variables 
$x_{p a}$ and represents a group treatment pattern of patients. Such a pattern of patient group treatments will be termed a column for the remainder of the paper. Every feasible column has to satisfy the bed capacity constraint, the OR time constraint and the constraint on the number of admitted patients.

Variables $x_{p a}$ can be aggregated at different levels, such as wards, surgeon groups and patient groups, providing us with alternative decomposition methods (Winston 1997; Beliën and Demeulemeester 2007). For instance, the aggregation of a set of variables $x_{p a}$ for all active days $a$ and for all patient groups $p$ of ward $w$ will form a new decision variable representing a possible pattern of patient group treatments for ward $w$. The level of aggregation is termed a decomposition unit throughout the rest of the paper. By finding a feasible column combination that covers the whole hospital and generates the highest profits, we obtain an optimal case mix pattern, together with an assignment scheme of beds and OR blocks.

The idea of enumerating all feasible columns is intimidating, since it indeed is highly time-consuming. Fortunately, the branch-and-price approach does not require a complete enumeration. Once provided with a pool of initial columns to start with, it has a smart scheme to find new columns and, moreover, only favorable columns will be generated and added, which limits the computation time significantly.

The original ILP model $(1-7)$ can be decomposed on different units, which may result in different reformulations. The choice of decomposition unit only affects the definition of the new decision variable, while both the objective of the profit maximization and the constraints on the existing resource capacity and on the number of the admitted patients within a cycle length remain unchanged. Subsequently, three decomposition based reformulations are presented respectively.

\subsubsection{Decomposition on Wards}

Decomposition on wards means that a set of variables $x_{p a}$ for all active days $a$ and for all patient groups $p$ whose patients are transferred to ward $w$ will be aggregated to form a feasible column for each ward $w$. To represent these columns, a new binary decision variable is introduced and defined as follows:

$$
c_{w k}= \begin{cases}1, & \text { if column } k \text { is selected for ward } w \\ 0, & \text { otherwise }\end{cases}
$$


Besides, the new sets, indices, variables and coefficients needed are given below:

$K$ : set of feasible columns with index $k$;

$y_{w k}$ : the number of beds allocated to ward $w$ if column $k$ is selected for ward $w$;

$z_{w a k}:$ the number of blocks assigned to surgeon groups of ward $w$ on day $a$ if selecting column $k$;

$r_{w k}$ : the total profits generated by treating patients of ward $w$ if column $k$ is selected for ward $w$.

Then, the case mix problem can be reformulated as follows:

$$
\begin{array}{ll}
\max & \sum_{w \in W} \sum_{k \in K} r_{w k} c_{w k} \\
\text { s.t. } & \sum_{w \in W} \sum_{k \in K} y_{w k} c_{w k} \leq B E D S, \\
& \sum_{w \in W} \sum_{k \in K} z_{w a k} c_{w k} \leq B L O C K S, \quad \forall a \in A, \\
& \sum_{k \in K} c_{w k}=1, \quad \forall w \in W, \\
& c_{w k} \in\{0,1\}, \quad \forall w \in W, k \in K .
\end{array}
$$

The objective function (8) maximizes the sum of the generated profits over all wards. Constraint (9) indicates the bed capacity limit for all wards. Constraint set (10) indicates the OR blocks limit on each active day. Constraints (11) and (12) imply that only one column is selected for every ward.

Clearly, the formulation above takes on a more compact form than the original ILP model. However, a further look at the new model raises the concern that the number of possible treatment patterns for each ward can be so numerous that enumerating all columns is practically impossible. Even if the enumeration is feasible for small problem dimensions, the huge number of integer variables can also make the problem unsolvable. In order to circumvent the formidable task of total enumeration, the technique of column generation is adopted, which is well suited to tackle this kind of problems. With column generation, the columns do not have to be enumerated from the very beginning, but are iteratively added after careful generation and selection. Moreover, at each step, we do not simply find and add a feasible column, but focus only on those with favorable reduced costs. The column generation process is repeated continuously 
until no more favorable columns are found. This technique can effectively limit the total number of column variables produced and thus speed up the whole computational process.

In order to complete the problem reformulation, the column pricing problem also needs to be defined. The formulation above $(8-12)$ that retains the target of profit maximization is called the master problem, while the pricing step is called the subproblem for the rest of the paper. In addition, the master problem containing only part of the columns is called the restricted master problem, while the master problem with the relaxed variables $\left(c_{w k} \in[0,1]\right)$ is called the linear relaxed master problem. In order to formulate the pricing subproblem, more required variables and coefficients are listed as follows:

$\pi$ : the dual price of the bed capacity allocation constraint (9);

$\lambda_{a}$ : the dual price of the OR blocks assignment constraint on active day $a(10)$;

$\gamma_{w}:$ the dual price of the one-column selection constraint for ward $w(11)$

$x_{p a k}$ : the number of patients of group $p$ that receive surgery on active day $a$ according to column $k$;

$z_{\text {sak }}$ : the number of blocks assigned to surgeon group $s$ on active day $a$ according to column $k$.

Then, the reduced cost of column $k$ for ward $w$ can be expressed by

$$
R C_{w k}=r_{w k}-y_{w k} \pi-\sum_{a \in A} z_{w a k} \lambda_{a}-\gamma_{w}=\sum_{p \in P_{w}} r_{p} \sum_{a \in A} x_{p a k}-y_{w k} \pi-\sum_{a \in A} z_{w a k} \lambda_{a}-\gamma_{w}
$$

For a maximization problem, when the reduced cost of column $k$ is positive, this column is favorable and thus should be added to the column pool of the master problem. Otherwise, the column should be discarded and kept out of the computational process. Hence, by finding the maximum value of the reduced costs for all possible columns, we get to know whether there still exist favorable columns to be added to the master problem. Thus, the subproblem pricing out a favorable column $k$ for ward $w$ can be formulated as below:

$$
\begin{aligned}
& \max \sum_{p \in P_{w}} r_{p} \sum_{a \in A} x_{p a k}-y_{w k} \pi-\sum_{a \in A} z_{w a k} \lambda_{a} \\
& \text { s.t. } \sum_{p \in P_{w}} \sum_{a \in A_{p d}} x_{p a k} \leq y_{w k}, \quad \forall d \in D,
\end{aligned}
$$




$$
\begin{aligned}
& z_{w a k}=\sum_{s \in S_{w}} z_{s a k}, \quad \forall a \in A, \\
& \sum_{p \in P_{s}} d u r_{p} x_{p a k} \leq z_{s a k} L E N G T H, \quad \forall s \in S_{w}, a \in A, \\
& L B_{p} \leq \sum_{a \in A} x_{p a k} \leq U B_{p}, \quad \forall p \in P_{w}, \\
& x_{p a k}, y_{w k}, z_{w a k}, z_{s a k} \in \mathbb{Z}^{+}, \quad \forall s \in S_{w}, p \in P_{w}, a \in A .
\end{aligned}
$$

The objective function (13) aims to maximize the variable part of the reduced cost. Constraint set (14) establishes that the allocated bed capacity is the maximum daily bed requirement for ward $w$. Equality set (15) calculates the total number of OR blocks assigned to the surgeon groups of ward $w$ on each active day $a$. Constraint set (16) indicates that the total surgery time for a surgeon group on an active day cannot exceed its assigned operating room time. The patient admission volume constraints (17) and the integer variable constraint (18) are imposed as well.

The constant part $\gamma_{w}$ of the reduced cost is removed from the objective function as it is determined by the solution of the restricted master problem. When the reduced cost for all wards $w$ is non-positive, no more favorable columns exist and the linear relaxed master problem is solved optimally.

\subsubsection{Decomposition on Surgeon Groups}

If the decomposition is based on the surgeon groups, a set of variables $x_{p a}$ for all active days $a$ and for all patient groups $p$ whose patients are operated on by surgeon group $s$ are aggregated to form a column for surgeon group $s$. Similarly, a new binary decision variable is introduced to represent these columns:

$$
c_{s k}= \begin{cases}1, & \text { if column } k \text { is selected for surgeon group } s \\ 0, & \text { otherwise }\end{cases}
$$

Also, the needed new variables and coefficients consist of:

$y_{s d k}$ : the number of beds occupied on day $d$ by patients of surgeon group $s$ if column $k$ is selected;

$r_{s k}$ : the total profits generated by treating patients of surgeon group $s$ if column $k$ is selected.

Then, the master problem is formulated as below: 


$$
\begin{aligned}
\max & \sum_{s \in S} \sum_{k \in K} r_{s k} c_{s k} \\
\text { s.t. } & \sum_{w \in W} y_{w} \leq B E D S, \\
& \sum_{s \in S_{w}} \sum_{k \in K} y_{s d k} c_{s k} \leq y_{w}, \quad \forall w \in W, d \in D, \\
& \sum_{s \in S} \sum_{k \in K} z_{s a k} c_{s k} \leq B L O C K S, \quad \forall a \in A, \\
& \sum_{k \in K} c_{s k}=1, \quad \forall s \in S, \\
& y_{w} \in \mathbb{Z}^{+}, c_{s k} \in\{0,1\}, \quad \forall w \in W, s \in S, k \in K .
\end{aligned}
$$

The objective function (19) aims at maximizing the total profits generated over all surgeon groups. Constraint (20) indicates the bed capacity allocation, and constraint set (21) guarantees that the daily bed occupancy of each ward cannot exceed its assigned quota. Constraint set (22) demonstrates the OR blocks restriction on each active day. Constraints (23) denote the one-column selection for every surgeon group $s$, and the last constraint (24) exhibits the binary or integer property of the variables.

To construct the pricing subproblem, more required parameters are introduced:

$\pi_{w d}$ : the dual price of the bed occupancy constraint of ward $w$ on day $d(21)$;

$\lambda_{a}$ : the dual price of the OR blocks assignment constraint on active day $a(22)$;

$\gamma_{s}$ : the dual price of the one-column selection constraint for surgeon group $s$ (23).

Then, the reduced cost of column $k$ for surgeon group $s$ is expressed by the following formula:

$$
R C_{s k}=r_{s k}-\sum_{d \in D} y_{s d k} \pi_{w d}-\sum_{a \in A} z_{s a k} \lambda_{a}-\gamma_{s}=\sum_{p \in P_{s}} r_{p} \sum_{a \in A} x_{p a k}-\sum_{d \in D} y_{s d k} \pi_{w d}-\sum_{a \in A} z_{s a k} \lambda_{a}-\gamma_{s} .
$$

Thus, the subproblem pricing out favorable column $k$ for surgeon group $s$ can be written as:

$$
\begin{aligned}
& \max \sum_{p \in P_{s}} r_{p} \sum_{a \in A} x_{p a k}-\sum_{d \in D} y_{s d k} \pi_{w d}-\sum_{a \in A} z_{s a k} \lambda_{a} \\
& \text { s.t. } \sum_{p \in P_{s}} \sum_{a \in A_{p d}} x_{p a k}=y_{s d k}, \quad \forall d \in D,
\end{aligned}
$$




$$
\begin{aligned}
& \sum_{p \in P_{s}} d u r_{p} x_{p a k} \leq z_{\text {sak }} L E N G T H, \quad \forall a \in A, \\
& L B_{p} \leq \sum_{a \in A} x_{p a k} \leq U B_{p}, \quad \forall p \in P_{S} \\
& x_{p a k}, y_{s d k}, z_{s a k} \in \mathbb{Z}^{+}, \quad \forall p \in P_{s}, a \in A, d \in D .
\end{aligned}
$$

The objective function (25) maximizes the variable part of the reduced cost $R C_{s k}$. Constraint set (26) calculates the daily bed occupancy of patients treated by surgeon group $s$. Constraint set (27) ensures that the total surgery time of surgeon group $s$ on an active day cannot exceed its assigned operating room time. Also, the patient admission volume constraints (28) and the integer variable constraint (29) are displayed. When no more favorable columns could price out for any surgeon group, the relaxed master problem is solved optimally with the column generation method.

\subsubsection{Decomposition on Patient Groups}

When the decomposition unit is set as the patient group, a set of variables $x_{p a}$ for all active days $a$ and for a patient group $p$ are aggregated to form a feasible column for this patient group $p$, which is expressed by the defined binary column variable:

$$
c_{p k}= \begin{cases}1, & \text { if column } k \text { is selected for patient group } p \\ 0, & \text { otherwise }\end{cases}
$$

The needed other variables and coefficients are described as follows:

$y_{p d k}$ : the number of beds occupied by patients of group $p$ on day $d$ if column $k$ is selected;

$z_{\text {pak }}:$ the number of blocks required by patients of group $p$ on active day $a$ if selecting column $k$;

$r_{p k}$ : the profits generated by treating patients of group $p$ if column $k$ is selected for patient group $p$.

After introducing the required new variables, the master problem is formulated as below:

$$
\begin{aligned}
& \max \sum_{p \in P} \sum_{k \in K} r_{p k} c_{p k} \\
& \text { s.t. } \sum_{w \in W} y_{w} \leq B E D S,
\end{aligned}
$$




$$
\begin{aligned}
& \sum_{p \in P_{w}} \sum_{k \in K} y_{p d k} c_{p k} \leq y_{w}, \quad \forall w \in W, d \in D, \\
& \sum_{s \in S} z_{s a} \leq \text { BLOCKS, } \forall a \in A, \\
& \sum_{p \in P_{s}} \sum_{k \in K} z_{p a k} c_{p k} \leq z_{s a}, \quad \forall s \in S, a \in A, \\
& \sum_{k \in K} c_{p k}=1, \quad \forall p \in P, \\
& c_{p k} \in\{0,1\}, \forall p \in P, k \in K ; \quad y_{w}, z_{s a} \in \mathbb{Z}^{+}, \forall w \in W, s \in S, a \in A .
\end{aligned}
$$

The objective function (30) maximizes the total profits generated over all patient groups. Constraints (31) and (32) regulate the allocation and utilization of the bed capacity. Constraint sets (33) and (34) refer to the OR blocks assignment and their utilization on each active day. Constraints (35) and (36) indicate the one-column selection for each patient group and the binary/integer variables.

Similar with the above two reformulations, after introducing the dual variables $\pi_{w d}, \lambda_{s a}$ and $\gamma_{p}$ for constraints (32), (34) and (35) respectively, the reduced cost of column variable $c_{p k}$ can be figured out:

$$
R C_{p k}=r_{p k}-\sum_{d \in D} y_{p d k} \pi_{w d}-\sum_{a \in A} z_{p a k} \lambda_{s a}-\gamma_{p}=\sum_{a \in A} r_{p} x_{p a k}-\sum_{d \in D} y_{p d k} \pi_{w d}-\sum_{a \in A} z_{p a k} \lambda_{s a}-\gamma_{p} .
$$

And then, the pricing subproblem is completed as:

$$
\begin{array}{ll}
\max & \sum_{a \in A} r_{p} x_{p a k}-\sum_{d \in D} y_{p d k} \pi_{w d}-\sum_{a \in A} z_{p a k} \lambda_{s a} \\
\text { s.t. } & \sum_{a \in A_{p d}} x_{p a k}=y_{p d k}, \quad \forall d \in D, \\
& x_{p a k} d u r_{p}=z_{p a k} L E N G T H, \quad \forall a \in A, \\
& L B_{p} \leq \sum_{a \in A} x_{p a k} \leq U B_{p}, \\
& z_{p a k} \geq 0, x_{p a k}, y_{p d k} \in \mathbb{Z}^{+}, \quad \forall a \in A, d \in D .
\end{array}
$$

The objective function (37) maximizes the variable part of the reduced cost $R C_{p k}$. Constraints (38-40) calculate the daily bed occupancy, the OR blocks required on each active day and the cyclic admission 
volume of patient group $p$ respectively. Constraint (41) shows whether the variables $x_{p a k}, y_{p d k}$ and $z_{p a k}$ are integer or not.

Nevertheless, a further look at the above model reveals an inherent pitfall that prevents it from being solved by column generation. In a branch-and-price approach, the integer variables of the master problem are relaxed and branching is performed on fractional solutions to derive an integer feasible solution. Once an integer solution is obtained for the binary column variables, the integrality of all other integer variables is ensured automatically. However, this model fails to satisfy this crucial requirement, since $z_{s a}$ cannot be guaranteed as an integer due to the continuous $z_{p a k}$. Hence, the decomposition model on patient groups cannot be solved with the branch-and-price approach.

\subsection{Branch-and-price Implementation}

In this section, the implementation of the branch-and-price approach is specified. The principle of branchand-price is explained through a diagram, followed by the detailed descriptions of how the key steps, such as column initialization, pricing and branching, are carried out.

\subsubsection{Branch-and-price Overview}

The general scheme of the branch-and-price approach is illustrated in Figure 2. The algorithm starts with a pool of initial columns, which are usually generated through heuristic methods and used to initialize the computation. In addition, super columns are also included in the restricted master problem to ensure the solution feasibility at each level of the branch-and-bound tree. At each round, the relaxed master problem is solved with the column generation method. The dual prices are then obtained and transferred to the pricing subproblem. The restricted master problem is repeatedly updated with new columns and is solved until no more favorable columns are produced. If the solution of the linear relaxed master problem satisfies integrality, an integer feasible solution is found. Otherwise, the master problem will be further constrained with new branching conditions and solved again. Any integer solution to the master problem is recognized as a lower bound and the optimal solution to the relaxed problem is identified as an upper 
bound on the optimal objective value. The best bounds will be checked against the newly generated ones and be updated if necessary. The iteration loop continues until the branch-and-bound tree is thoroughly explored or when the best upper bound and the best lower bound are identical.

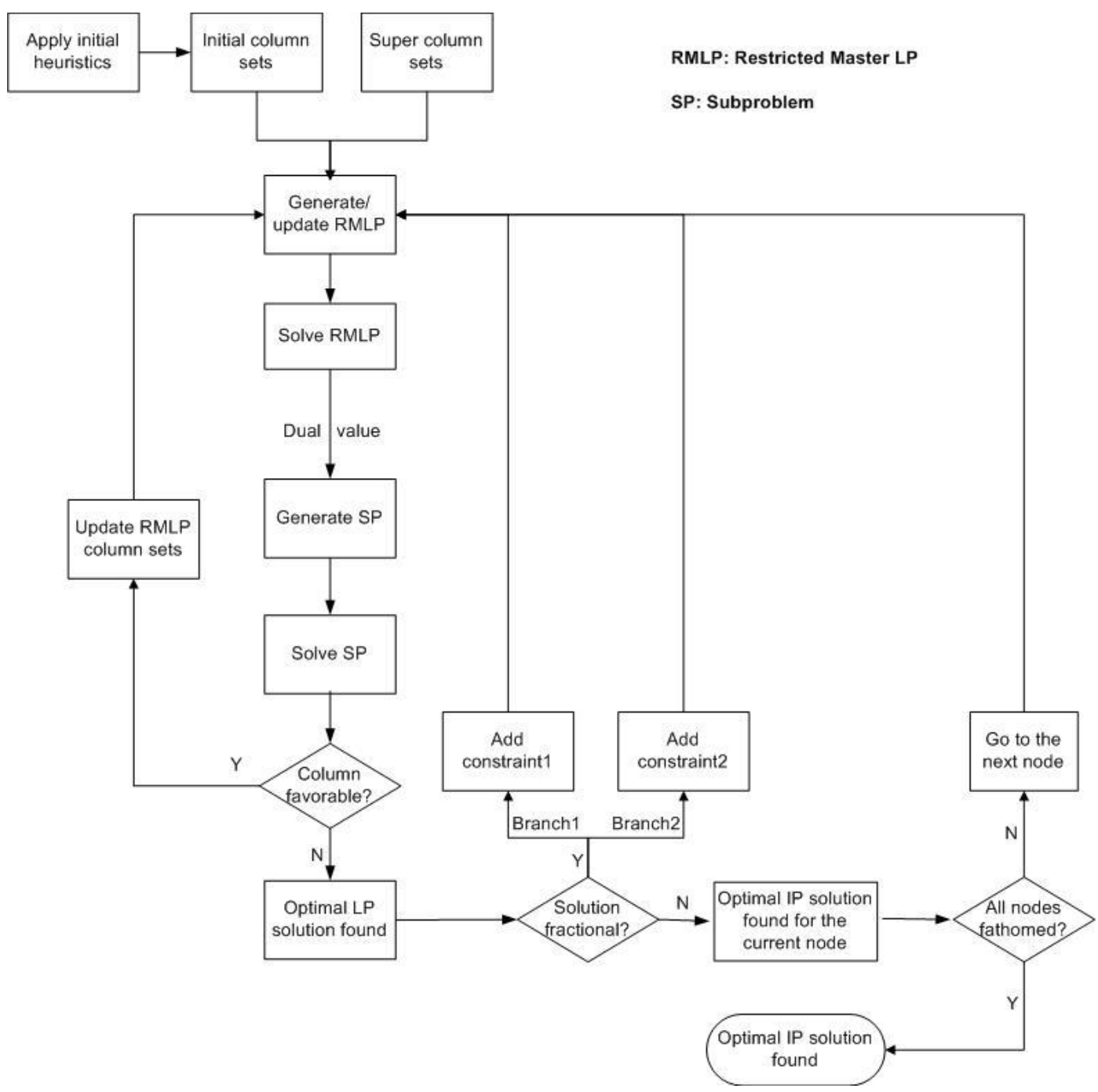

Figure 2: Branch-and-price Scheme

\subsubsection{Column Initialization}

The generation of initial columns is combined with a problem feasibility check. In order to verify whether the problem is feasible, the numerical range (6) of patient admissions of each group is modified into the fixed lower bound in the original ILP model:

$$
\sum_{a \in A} x_{p a}=L B_{p}, \quad \forall p \in P .
$$


Clearly, the feasible solution of the reduced model is also feasible to the original ILP model. However, if the reduced model has no feasible solution, it means that the given capacity cannot satisfy the minimum requirement of patient admissions and thus the problem is infeasible. Actually, after fixing the number of admitted patients of each group to its lower bound, the original problem is simplified into a resourceconstrained assignment problem, and thereby it can be solved easily. Moreover, the thus found solution can generate the feasible columns for the master problem. To find more initial columns, the admission volume for a certain patient group $p$ is relaxed to its upper bound (i.e., $\leq U B_{p}$ ), while the number of admitted patients for all other groups is kept at their lower bound, and then we solve the revised problem. Repeatedly applying this procedure for all patient groups, more feasible columns are produced and added to the initial column pool.

\subsubsection{Pricing out}

In our research, the pricing subproblem is solved with the commercial ILP software ILOG CPLEX. The major concern in this step is to determine which and how many favorable columns are produced and are to be added to the master problem at each round of the linear optimization. In theory, any column with a positive reduced cost prices out for a maximization problem and thus can be added to the master problem. However, on the one hand, adding as many favorable columns as possible at a time enlarges the column pool too rapidly. On the other hand, the total number of favorable columns can be so gigantic that finding them all becomes practically infeasible, especially at an early computing stage. Furthermore, introducing more columns expands the solution space to be searched and thus increases the risk of slowing down the solution of the master problem.

An alternative approach is to add only one or a few columns at a time. In our work, we choose to add one favorable column for each decomposition unit because finding the best column is neither always easy nor necessary. In practice, any favorable column will enable the computation to continue. Therefore, we impose a time limit in column searching. If no favorable column is found, more time will be allocated to the subproblem. Otherwise, the best column found within the time limit will be retrieved for use. 


\subsubsection{Branching}

More often than not, the linear relaxed master problem ends up with a fractional solution, which brings up the necessity of branching. However, applying a standard branch-and-bound procedure to the restricted master problem will not guarantee an optimal or even feasible solution. Therefore, new favorable columns should be generated and added to the column pool after branching, and the branching strategy should not increase the difficulty of solving the pricing subproblem.

Clearly, branching on the column variable $c_{* k}$ will produce an uneven partition of the solution space, and also destroys the structure of the pricing problem. Otherwise, branching on the case mix variable $x_{p a}$ cannot effectively separate the solution space. Actually, two different case mix patterns might correspond to an identical resource allocation scheme and generate equal profits, which results in the same column in the master problem. Hence, we adopt the branching strategy based on the capacity assignment variables, that is, $y_{w}$ and $z_{w a}$ for the decomposition model on wards, or $y_{s d}$ and $z_{s a}$ for the decomposition model on surgeon groups. If the solved optimal solution to the relaxed master problem is not integral, there exist at least two fractional column variables related to one ward $w$, for instance $c_{w k}$ and $c_{w k^{\prime}}$, and there exists at least one value of the capacity assignment variables $\left(y_{w}\right.$ and $\left.z_{w a}\right)$ different from each other in these two columns, say $z_{w a k}<z_{w a k^{\prime}}$. Thus, the branching condition can be defined as

$$
z_{w a} \leq z_{w a k} \text { and } z_{w a} \geq z_{w a k}+1 .
$$

Branching on the capacity assignment variables not only excludes the fractional optimal solution effectively, but also partitions the solution space into two branches more evenly. Moreover, the pricing subproblem is not changed structurally and is still tractable after the branching. In fact, only the feasible bound of the capacity assignment variables is altered after adding the branching constraints.

\subsection{Acceleration Techniques}

The integrality of a branch-and-price algorithm is ensured by a proper column generation method together with an effective branching scheme. Moreover, introducing one or more acceleration techniques would further enhance the performance of the algorithm. 


\subsubsection{Lagrangian Bound}

A complete computation process can be divided into two parts: searching for the optimal solution and proving its optimality. The fact that the time spent on proving optimality may be comparable or even considerably longer than finding the optimal solution is termed as the tailing-off effect. Applying a Lagrangian bound can help to check whether the current node in the branch-and-bound tree possesses the potential to outperform the present best bound. If the answer is no, the node is fathomed without further computation. This early termination of nodes saves the computation time without any risk of missing the optimal solution (van den Akker et al. 2002).

\subsubsection{Active Generation of Integer Feasible Solutions}

Two kinds of integer problems are solved in our branch-and-price approach. The first one is the pricing subproblem. The second one is not an essential part of the branch-and-price algorithm but is specifically introduced to find an integer feasible solution and to provide a lower bound on the optimal value. In order to improve the lower bound more quickly and to terminate the computation process earlier, we do not just passively wait for the linear relaxed master problem to produce an integer feasible solution, but actively seek for some ones as well. At the end of each round of the linear optimization when no more favorable columns price out, the master problem is solved again with unrelaxed integer constraints. The unrelaxed master problem, if feasible, provides us a lower bound on the optimal value. The best lower bound will be checked against the latest bound found and be updated if necessary.

\subsubsection{Cutoff Value}

When solving the integer problems, a cutoff value (if readily available) can be specified to help terminate the calculation earlier. If the objective value of the integer problem is recognized as impossible to exceed the cutoff value, there is no need to continue searching for the optimal solution or any feasible solutions. A rough judgement of whether the objective value can beat the cutoff value is often less time-consuming than to obtain an optimal or feasible solution. Hence, applying a cutoff value will help to accelerate the computation process. Recall the fact that the reduced cost $R C_{* k}$ of each column within the decomposition 
based models is divided into a variable part and a constant part, and that only the column with a positive reduced cost can be generated for the master problem. Thus, the constant part can be set as a cutoff value for the pricing subproblem since its objective function addresses the variable part of the reduced cost only. Similarly, when solving the unrelaxed master problem, the cutoff value adopted is set as the best lower bound that has been found so far.

\section{Computational Results}

In this section, the case mix problem is solved with two exact methods proposed in this research. The ILP solver ILOG CPLEX is used to solve the original ILP formulation, and the branch-and-price algorithms are deployed to solve the reformulated models. Firstly, the computational results of the ILP solver solving the original ILP model are presented, which clearly illustrates that the commercial software fails to solve the real case mix problem effectively. Secondly, the computational results of the ward-decomposed and surgeon group-decomposed methods are compared and analyzed in terms of efficiency and effectiveness. Nevertheless, as mentioned before, the decomposition model on patient groups cannot be solved with the branch-and-price approach and thus its computational results are not available here. Finally, the relative performance and behavior of the two solvable decomposition methods are discussed intensively through further numerical experiments.

All experiments are performed on a personal computer equipped with a Duo Core CPU 3.16GHz and a Windows XP operating system. The algorithms are coded in Microsoft Visual Studio 2005 Version 8.0 and linked with the ILOG CPLEX 10.2 optimization library for solving the master and subproblems in the branch-and-price approaches.

\subsection{ILP Method}

The original ILP model $(1-7)$ is solved with the commercial software ILOG CPLEX 10.2 with standard settings. To study the impact of the problem dimensions on the performance of the software, we start with a number of instances with dummy data that involve no more than 5 departments, although in reality it is 
common for a hospital to contain 25 or more departments. The testing results of the small problems infer that the commercial software will fail to solve the real cases.

Without loss of generality, it is assumed that each department has three surgeon groups with each handling 3 pathologies in the testing instances. Moreover, for each patient group, the lower bound on the number of admitted patients per cycle is set as one, while the upper bound is generated randomly from a discrete uniform probability distribution within the interval $[3,6]$. Similarly, other data parameters are also generated from discrete uniform distribution functions. Specifically, the average LOS per pathology is distributed in the interval $[4,10]$, the expected surgery duration is distributed in the interval $[60,240]$, and the mean treatment reward is distributed in the interval $[12,30]$. In addition, the total number of beds and the number of operating rooms are fixed as different amounts in various instances. Table 1 displays the computational results of three problem instances.

Table 1: Computational Results of the ILP Solver

\begin{tabular}{|c|c|c|c|c|c|c|}
\hline Dept. No. ${ }^{I}$ & Best $\operatorname{sln}^{2}$ & Optimal? & $L P$ relaxation & Nodes expl. ${ }^{3}$ & Nodes left & Comp. time $(s)^{4}$ \\
\hline 1 & 240 & $\mathrm{Y}$ & 240 & 38 & 0 & 0.015 \\
\hline 3 & 680 & $\mathrm{Y}$ & 681 & 159600 & 15 & 49.297 \\
\hline 5 & 1357 & $\mathrm{~N}$ & 1629.7 & 1498900 & 1465879 & 1800 \\
\hline
\end{tabular}

Note. 1, the number of departments (or wards); 2, sln.: solution; 3, expl.: explored; 4, the computation time in seconds.

In Table 1, a series of performance indicators are listed, which consist of the best solution found, whether the optimality is proven, the LP relaxation, the number of explored nodes and left nodes, and the computation time. Clearly, the computation time of the ILP solver exhibits an obvious increasing trend with the problem dimension. In the presence of only one ward, the solver obtains the optimal solution with proven optimality instantly. When the number of wards increases to 3 , the computing process slows down to 49 seconds. In the case of five wards, a much smaller dimension than real-life cases, the ILP optimizer fails to find the optimal solution within the prescribed time limit of 1800 seconds. To further test the efficiency of the ILP method, the computation time limit is extended to 7200 seconds for the five- 
ward instance. A higher objective value of 1412 (as compared to 1357) is reached after 7200 seconds, but the optimal solution is still not found. At this time, the branch-and-cut tree size already exceeds $700 \mathrm{MB}$ and the node file is over $600 \mathrm{MB}$ after CPLEX ran for two hours. Actually, experiments show that the computation time is not the single barrier for finding the optimal solutions. For the five-ward instance, CPLEX runs out of memory after five hours without reaching the optimal value.

Therefore, it can be inferred that the ILP method is not efficient enough to give a satisfactory result for larger problem dimensions. However, the proposed branch-and-price algorithms successfully exhibit their efficiency and effectiveness to solve the case mix problem, as will be illustrated in the next section.

\subsection{Branch-and-price Algorithms}

In this section, the decomposition model on wards and the decomposition model on surgeon groups are solved with the branch-and-price approach. Their computational results are presented and compared, and the performances of the two algorithms are analyzed in detail.

In order to demonstrate the efficiency and effectiveness of the developed branch-and-price algorithms, the computational results of seven instances with different problem dimensions are exhibited in Table 2. These seven problem instances comprise the three small problems used to test the ILP method before as well as four medium and large size new problems. Similarly, it is assumed that each ward accommodates the patients of three surgeon groups with each treating three pathologies, and the total number of beds and the number of OR blocks are set at different levels for every instance. In addition, the needed treatment parameters are generated on the basis of discrete uniform probability distributions as above.

Besides the data parameters including the number of wards, beds and OR blocks of each instance, the performance indicators of the two algorithms are presented in Table 2, which consist of the best solution found, the computation time and the number of explored nodes and left nodes. From the table, we can see that in general the computation time increases with problem dimension. For all the tested instances, the ward-decomposed method finds the optimal solution very quickly and proves its optimality with only a few nodes explored. However, the surgeon group-decomposed method can only find the optimal solution 
quickly for the small problems, while it fails to find the optimal solution within the prescribed time limit for the larger cases. Moreover, the best solution found is usually 5 to 10 percent lower than the optimum, and the efficiency drastically decreases as the problem dimension grows.

Table 2: Computational Efficiency and Effectiveness of Branch-and-price

\begin{tabular}{ccccccccc}
\hline \multicolumn{1}{l}{ Parameters } & \multicolumn{5}{c}{ Ward-decomposed } & \multicolumn{5}{c}{ Surgeon group-decomposed } \\
\hline wards & beds & blocks & Best sln. & Comp. time & Nodes(E/L) & Best sln. & Comp. time & Nodes $(E / L)^{*}$ \\
\hline 1 & 8 & 1 & 240 & 0.266 & $1 / 0$ & 240 & 3.218 & $24 / 17$ \\
3 & 25 & 2 & 680 & 4.687 & $10 / 0$ & 680 & 131.685 & $436 / 417$ \\
5 & 50 & 3 & 1471 & 3.048 & $1 / 0$ & 1471 & 162.937 & $240 / 241$ \\
10 & 100 & 8 & 3376 & 6.436 & $1 / 0$ & 3122 & 3600 & $354 / 355$ \\
15 & 150 & 10 & 4776 & 9.233 & $1 / 0$ & 4548 & 3600 & $348 / 349$ \\
20 & 180 & 13 & 5745 & 9.812 & $1 / 0$ & 5342 & 3600 & $346 / 347$ \\
25 & 220 & 18 & 7319 & 20.87 & $7 / 0$ & 6796 & 3600 & $350 / 351$ \\
\hline
\end{tabular}

Note. *the number of explored nodes/left nodes

In contrast to the ward-decomposed method, the surgeon group-decomposed method suffers from two factors: a slow searching process for the optimal solution and a time-consuming process of optimality testing. The former can be attributed to the large number of pricing steps involved at each iteration and to the large number of variables generated. As our test data assume that the surgeon groups outnumber the wards by a factor of three, the number of subproblems to be solved at each round of the surgeon groupdecomposed method is three times more than that of the ward-decomposed method. In addition, as in each iteration of the column generation process one column will be generated for each decomposition unit and one ward corresponds to several surgeon groups, the surgeon group-decomposed method generally produces more variables than the ward-decomposed method, which will make the master problem more difficult to be solved. Moreover, it is observed that the time needed to explore one node increases significantly with the problem size. For the surgeon group-decomposed method, one node is usually explored within 1 second when there are only five wards, but it expands to more than 10 seconds on the 
average for the cases of ten wards or more. For the same reason, it is not necessarily better to start out with more initial columns. Clearly, a large number of columns might enable one to find the first good quality feasible solution rather quickly, but meanwhile can also limit the general computation speed.

The second factor, the long optimality testing, can be explained by the weak relaxation bound of the surgeon group-decomposed method. Table 3 compares the quality of the relaxation efficiency of the two decomposition methods. The relaxation efficiency is defined as the difference between the LP relaxation value and the optimal value over the optimum. From Table 3, we can see that the relaxation efficiency of the surgeon group-decomposed method mostly falls into the range of 5-10\%, while the ward-decomposed method gives a much tighter bound (usually within $0.01 \%$ for the tested data). Hence, even if the optimal solution might be sought out within a short time, a loose relaxation bound will still impose a demand for a long optimality testing, which prolongs the whole computational process in general.

Table 3: Comparison of the Relaxation Efficiency of Two Decomposition Methods

\begin{tabular}{lccccccc}
\hline \multicolumn{2}{l}{ Parameters } & \multicolumn{3}{l}{ Relaxation bound } & \multicolumn{2}{l}{ Relaxation efficiency } \\
\hline wards & beds & blocks & Optimum & Ward-D* & Surg-D* & Ward-D & Surg-D \\
\hline 5 & 50 & 3 & 1471 & 1471.0 & 1531.0 & $0.00 \%$ & $4.08 \%$ \\
10 & 100 & 8 & 3376 & 3376.0 & 3516.0 & $0.00 \%$ & $4.15 \%$ \\
15 & 150 & 10 & 4776 & 4776.0 & 5142.0 & $0.00 \%$ & $7.66 \%$ \\
20 & 180 & 13 & 5745 & 5745.0 & 6354.0 & $0.00 \%$ & $10.60 \%$ \\
25 & 220 & 16 & 6947 & 6947.0 & 7683.0 & $0.00 \%$ & $10.59 \%$ \\
25 & 220 & 18 & 7319 & 7319.0 & 7891.0 & $0.00 \%$ & $7.82 \%$ \\
25 & 220 & 20 & 7570 & 7570.0 & 8003.0 & $0.00 \%$ & $5.72 \%$
\end{tabular}

Note. ${ }^{*}$ Ward-D: Ward-decomposed, Surg-D: Surgeon group-decomposed

Further analysis shows that the lower relaxation efficiency of the surgeon group-decomposed method comes from its loose variable bound in the problem formulation. When new columns are generated in the subproblem, the given resource capacity is used as the upper bound on the amount of consumed resources (i.e., $y_{w}, z_{w a}$ or $\left.y_{s d}, z_{s a}\right)$ by each decomposition unit. Clearly, by allowing a decomposition unit to access 
all the available resources, a quite loose variable bound might be produced, which will bring an extra area of the solution space to be explored even if the chance of finding the optimal or a feasible solution there is very small. Therefore, it can be inferred that a smaller decomposition unit (e.g., the surgeon group) is less likely to occupy all the resources, which tends to generate a looser variable bound and thus decreases the whole computation speed. In other words, the analysis above well supports the fact that the branch-andprice algorithm on the basis of the decomposition on wards produces a tighter master relaxation bound and runs at a faster speed.

Through the discussion on the computational results, the branch-and-price algorithm is illustrated to be extremely valuable in solving the studied case mix problem optimally. Moreover, the decomposition method on wards performs much better than the decomposition method on surgeon groups with respect to both the solution quality and the computation speed.

\section{Conclusions}

This paper describes a methodology for the case mix problem in the healthcare sector. In order to mitigate the increasing economic pressures, hospitals are expected to maximize their resource efficiency through choosing the optimal patient case mix pattern. With the objective of maximizing the total hospital profits, the case mix problem is formulated as an integer linear programming model to determine the optimal case mix selection and the corresponding resource capacity allocation, in which the case mix selection has to satisfy the resource capacity constraints and the patient admission limitation.

In order to solve the case mix problem optimally, two exact methods, ILP and branch-and-price, are proposed and deployed to work out the formulated ILP model. Through the discussion on the computing results, it is clearly demonstrated that the branch-and-price approach drastically outperforms the ILP method. In our numerical experiments, the commercial software ILOG CPLEX is applied to solve the ILP formulation directly, which exhibits that the computation time of ILOG CPLEX increases significantly as the problem dimension grows. In fact, the ILP solver can only figure out the very small sized problems 
efficiently, but fails to offer a high-quality integer feasible solution within a long computation time when the number of departments reaches or exceeds five.

However, the branch-and-price approach is investigated to be extremely speedy and effective when tackling the case mix problem at hand. From the column generation perspective, the original ILP model is reformulated according to the decomposition technique, so that the decomposed formulations are suitable to be solved within the framework of branch-and-price. Depending on the decomposition unit selected, three column generation based formulations are built, namely the decomposition model on wards, on surgeon groups and on patient groups respectively, but only the first two of them are able to be solved with branch-and-price algorithms. Moreover, for these two decomposed models, numerical results clearly indicate that the branch-and-price algorithm on the basis of the decomposition on wards performs much better than the one on surgeon groups in terms of both solution quality and computation speed. In addition, further analysis reveals that the choice of the decomposition unit has a significant impact on the efficiency and effectiveness of the branch-and-price algorithm.

Finally, it is worthy to be noted that, besides the profit maximization target, this work can be easily extended to other purposes. Actually, the reward coefficient in the objective function is not limited to the financial return but can be set to any other desired performance indicator in practice. On the other hand, this work adopts the unit perspective and focuses on the allocation and utilization of resources, but it would be interesting to introduce simulation technique to assess the performance of the capacity planning and to observe the service level of the treatment process from a chain perspective in order to enhance the ideal trade-offs between resource efficiency and patient service.

\section{Acknowledgements}

The authors acknowledge the support given to this project by the Fonds voor Wetenschappelijk Onderzoek (FWO)-Vlaanderen, Belgium under contract number G.0456.08. 


\section{References}

Adan, I.J.B.F., J.M.H. Vissers. 2002. Patient mix optimisation in hospital admission planning: A case study. International Journal of Operations \& Production Management 22(4) 445-461.

Baligh, H.H., D.J. Laughhunn. 1969. An economic and linear model of the hospital. Health Services Research 4(4) 293-303.

Bard, J.F., H.W. Purnomo. 2005. Preference scheduling for nurses using column generation. European Journal of Operational Research 164(2) 510-534.

Barnhart, C., E.L. Johnson, G.L. Nemhauser, M.W.P. Savelsbergh, P.H. Vance. 1998. Branch-and-price: Column generation for solving huge integer programs. Operations Research 46(3) 316-329.

Beliën, J., E. Demeulemeester. 2006. Scheduling trainees at a hospital department using a branch-andprice approach. European Journal of Operational Research 175(1) 258-278.

Beliën, J., E. Demeulemeester. 2007. On the trade-off between staff-decomposed and activitydecomposed column generation for a staff scheduling problem. Annals of Operations Research 155(1) 143-166.

Beliën, J., E. Demeulemeester. 2008. A branch-and-price approach for integrating nurse and surgery scheduling. European Journal of Operational Research 189(3) 652-668.

Blake, J.T., M.W. Carter. 2002. A goal programming approach to strategic resource allocation in acute care hospitals. European Journal of Operational Research 140(3) 541-561.

Blake, J.T., F. Dexter, J. Donald. 2002. Operating room managers' use of integer programming for assigning block time to surgical groups: A case study. Anesthesia and Analgesia 94(1) 143-148.

Blake, J.T., J. Donald. 2002. Mount Sinai hospital uses integer programming to allocate operating room time. Interfaces 32(2) 63-73.

Boldy, D. 1976. A review of the application of mathematical programming to tactical and strategic health and social services problems. Operational Research Quarterly 27(2) 439-448. 
Brandeau, M.L., D.S.P. Hopkins. 1984. A patient mix model for hospital financial planning. Inquiry 21(1) 32-44.

Carter, M.W. 2002. Diagnosis: Mismanagement of resources. ORMS Today 29(2) 26-32.

Dexter, F., J.T. Blake, D.H. Penning, D.A. Lubarsky. 2002a. Calculating a potential increase in hospital margin for elective surgery by changing operating room time allocations or increasing nursing staffing to permit completion of more cases: A case study. Anesthesia and Analgesia 94(1) 138-142.

Dexter, F., J.T. Blake, D.H. Penning, B. Sloan, P. Chuang, D.A. Lubarsky. 2002b. Use of linear programming to estimate impact of changes in a hospital's operating room time allocation on perioperative variable costs. Anesthesiology 96(3) 718-724.

Dowling, W.L. 1976. Hospital Production: A Linear Programming Model. Lexington Books, Lexington, MA.

Feldstein, M.S. 1967. Economic Analysis for Health Service Efficiency. North-Holland, Amsterdam.

Fetter, R.B., J.L. Freeman. 1986. Diagnosis related groups: Product line management within hospitals. The Academy of Management Review 11(1) 41-54.

Fetter, R.B., Y. Shin, J.L. Freeman, R.F. Averill, J.D. Thompson. 1980. Case mix definition by diagnosisrelated groups. Medical Care 18(2) 1-53.

Gemmel, P., R. Van Dierdonck. 1999. Admission scheduling in acute care hospitals: Does the practice fit with the theory? International Journal of Operations and Production Management 19(9) 863-878.

Hatcher, M.E., C. Connelly. 1988. A case mix simulation decision support system model for negotiating hospital rates. Journal of Medical Systems 12(6) 341-363.

Hughes, W.L., S.Y. Soliman. 1985. Short-term case mix management with linear programming. Hospital \& Health Services Administration 30(1) 52-60.

Kuo, P.C., R.A. Schroeder, S. Mahaffey, R.R. Bollinger. 2003. Optimization of operating room allocation using linear programming techniques. Journal of the American College of Surgeons 197(6) 889-895.

Mulholland, M.W., P. Abrahamse, V. Bahl. 2005. Linear programming to optimize performance in a department of surgery. Journal of the American College of Surgeons 200(6) 861-868. 
OECD. 2010. OECD Health Data 2010: Statistics and Indicators for 34 Countries. http://www.oecd.org/.

Rhyne, D.M., D. Jupp. 1988. Health care requirements planning: A conceptual framework. Health Care Management Review 13(1) 17-27.

Rifai, A.K., J.O. Pecenka. 1990. An application of goal programming in healthcare planning. International Journal of Operations \& Production Management 10(3) 28-37.

Robbins, W.A., N. Tuntiwongbiboon. 1989. Linear programming is a useful tool in case-mix management. Healthcare Financial Management 43(6) 114-116.

Roth, A.V., R. Van Dierdonck. 1995. Hospital resource planning: Concepts, feasibility, and framework. Production and Operations Management 4(1) 2-29.

Simon, H.A. 1960. The New Science of Management Decision. Harper \& Row, New York.

Smith-Daniels, V.L., S.B. Schweikhart, D.E. Smith-Daniels. 1988. Capacity management in health care services: Review and future research directions. Decision Sciences 19(4) 889-919.

Tingley, K.M., J.S. Liebman. 1984. A goal programming example in public health resource allocation. Management Science 30(3) 279-289.

van den Akker, M., H. Hoogeveen, S. van de Velde. 2002. Combining column generation and Lagrangean relaxation to solve a single-machine common due date problem. INFORMS Journal on Computing 14(1) $37-51$.

Vissers, J.M.H., I.J.B.F. Adan, J.A. Bekkers. 2005. Patient mix optimization in tactical cardiothoracic surgery planning: A case study. IMA Journal of Management Mathematics 16(3) 281-304.

Vissers, J.M.H., I.J.B.F. Adan, N.P. Dellaert. 2007. Developing a platform for comparison of hospital admission systems: An illustration. European Journal of Operational Research 180(3) 1290-1301.

Vissers, J.M.H., J.W.M. Bertrand, G. de Vries. 2001. A framework for production control in healthcare organizations. Production Planning \& Control 12(6) 591-604.

Winston, W.L. 1997. Operations Research: Applications and Algorithms (third edition). Duxbury Press, Belmont, CA. 